\title{
32. \\ Untersuchung über die Directrixen der Curven.
}

(Von Herrn L. Raabe zu Wien.)

\section{1.}

Nimmt man für eine gegebene ebene Curve einen Punct, der in der Ebene derselben sich befindet, als fix an, und nennt Radiusvector jede Gerade, die von diesem Puncte zu den Peripheriepuncten der Curve geführt wird, so wird jede andere Curve Directrix der gegebenen genannt, für welche die aus den Peripheriepuncten der gegebenen Curve an dieselbe g'ezogene Normalen, der Grölse nach den entsprechenden Radiivectoren gleich sind. So ist, wenn die Parabel als gegebene Curve und ihr Brennpunct als fix angesehen wird, jene auf der Axe der Parabel errichtete senkrechte Gérade, die aułserhalb der Parabel in der Entfernung des vierten Theils des Parameters von dem Scheitel fällt, die Directrix der Parabel. Diese Directrix für eine jede gegebene ebene Curve zu finden, und das Umgekehrte, nemlich für eine jede gegebene Directrix nebst fixem Punct, 'die entsprechende Curve zu finden, soll den Inhalt gegenwärtiger Untersuchung ausmachen.

Ist der erwähnte fixe Punct der Anfangspunct eines geradlinigen senkrechten Coordinatensystems, so sei die Gleichung der gegebenen Curve, auf dieses Coordinatensystem bezogen, von der Form:

$$
\text { (I) }{ }^{-} f(x, y)=0 \text {, }
$$

wo $x$ und $y$ die Coordinaten irgend eines Punctes der Peripherie der gegebenen Curve vorstellen. Trifft nun die aus diesem Puncte auf die zu suchende Directrix gefällte Normale, dieselbe in einem Puncte, dessen Coordinaten $\xi$ und $v$ gegen das. festgesetzte Coordinatensystem sind, so ist die Gleichung der Normale:

$$
(y-v) v^{\prime}+(x-\xi)=0
$$

wo $v^{\prime}$ den-Differentialcoefficienten $\frac{d v}{d \xi}$ der Iirectrix vorstellt.

Endlich mufs noch die Bedingung Statt haben, dafs die Gröfse der zwischen den beiden Puncten $x, y$ und $\xi, v$ enthaltenen Normale, gleich werden soll dem Radiusvector, oder der Geraden, die zwischen dem An- 
fangspunct der Coordinaten und dem Punct $x$, y enthalten ist. Es mufs daher noch folgende Gleichung:

$$
\text { (III) } \xi^{2}+v^{2}=2(\xi x+v y)
$$

Statt haben. Aus diesen drei Gleichungen die Coordinaten $x, y$ eines besonderen Punctes der Curve (I) eliminirt, erhält man eine Differentialgleichung der ersten Ordnung zwischen $\xi, v, v^{\prime}$, deren Integral die gesuchte Directrix geben wird.

\section{2.}

Wäre aber die Gleichung der Directrix gegeben und zwar von der Form:

$$
\text { (IV) } \varphi(\xi, v)=0
$$

auf dasselbe Coordinatensystem bezogen, von welchem wir im vorhergehenden Paragraph Gebrauch machten, so dürfte man nur, um die entsprechende Curve zu finden, aus der letzteren Gleichung den Werth von $v^{\prime}$ suchen und solchen in die Gleichung (II) substituiren, und dann vermittelst der so erhaltenen Gleichung (II) und der Gleichungen (III) und (IV), die Coordinaten $\xi$, $v$ eliminiren, wo dann als Resultat der Elimination die Endgleichung der entsprechenden Curve hervorgehen-würde.

\section{3.}

Man sieht aus dem Vorhergehenden, dars es keiné Schwierigkeiten hat, zu einer Directrix dịe entsprechende Curve für einen gegebenen fixen Punct zu finden, indem das ganze Verfahren auf einer einfachen Elimination beruht; hingegen hängt das Problem ,zu einer gegebenen Curve eine Directrix zu finden," wie bereits erwähnt wurde, von der Integration einer Differentialgleichung der ersten Ordnung ab, welche in sehr vielen Fällen unüberwindliche Hindernisse hat. Wir wollen daher bei sich darbietenden Problemen, statt der Integration nur das singuläre Integral der vorliegenden Differentialgleichung suchen, welches dieselben Dienste gewähren wird, wie das vollständige Integral. Denn es stelle

$$
F(\xi, v, a)=0
$$

das vollständige Integral der bereits erwähnten Differentialgleichung der Directrix vor, wo $a$ die durch Integration eingeführte willkürliche Constante ist. Für jeden besonderen Werth, den man der Constanten $a$ bei- legt, erhält man eine andere Directrix der Form und Lage nach; alle diese Directrixen aber werden den durch die Gleichungen (II) und (III) 
ausgesprochenen Bedingungen Genüge thun, daher auch die, sämmtliche Directrixen einhüllende Curve (da sie in jedem ihrer Puncte eine gemeinschaftliche Tangente, daher auch mit der ihr entsprechenden Directrix, eine gemeinschaftliche Normale hat) den Bedingungen (II) und (III) Genüge thun wird. Wir haben mithin nicht mehr nöthig, die Directrix selbst zu suchen, sondern nur die einhüllende Curve sämmtlicher Directrixen, die durch die Veränderlichkeit des Parameters $a$ entstehen. Nun stellt die aus einer Differentialgleichung zwischen zwei veränderlichen Gröfsen erhaltene singuläre Auflösung, nichts anderes als die Einhüllungscurve sämmtlicher Curven des vollständigen Integrals vor, die durch die Annahme, dafs ein Parameter $a$ veränderlich ist, entstehen. Daher reducirt sich das Verfahren auf folgendes. Nie Werthe für $x$ und $y$ aus den Gleichungen (II) und (III), nemlich:

$$
\text { (V) }\left\{\begin{array}{l}
x=\frac{\left(v^{2}-\xi^{2}\right) v^{\prime}+2 \xi v}{2\left(v-\xi v^{\prime}\right)} \\
y=\frac{\left(v^{2}-\xi\right)-2 \xi v v^{\prime}}{2\left(v-\xi v^{\prime}\right)},
\end{array}\right.
$$

substituire man in die Gleichung (I); dadurch erhält man eine Differentialgleichung von der Form:

$$
\psi\left(\xi, v, v^{\prime}\right)=0 .
$$

Für diese Gleichung suche man eine singuläre Auflösung; so stellt die so erhaltene Relation zwischen $\xi$ und $v$ eine Curve vor, welche die Eigenschaften, die wir an der Directrix gesucht haben, mit ihr gemein hat. - Die auf diesem Wege erhaltene Curve werden wie zum Unterschiede der eigentlichen Directrix, Curve der Directrixen nennen.

4.

Wir wollen das Vorhergehende auf einige besondere Fälle anwen. den. Es sei die gegebene Curve eine Isinie der zweiten Ordnung. Der fixe Punct sei" ein Brennpunct derselben, und die Axe der $x$ sei die gröIsere Hauptaxe dieser Linie. Dann hat man zur Gleichung derselben:

$$
f(x, y)=x^{2}+y^{2}-(p+e x)^{2}=0 \text {, }
$$

wo $p$ der halbe Parameter der Curve ist, und $e$ das Verhältnifs der Excentricität zur grofsen Axe. Setzt man in die letzte Gleichnng die Werthe für $x$ und $y$ aus den Gleichungen $(V)$, so ist die Differentialgleichung:

$\psi\left(\xi, v, v^{\prime}\right) \doteq\left\{2 p v+2 e \xi v-\left[2 p \xi-e\left(v^{2}-\xi^{2}\right)\right] v^{\prime}\right\}^{2}-\left(1+v^{\prime 2}\right)\left(\xi^{2}+v^{2}\right)^{2}=0$. 
Aus dieser Gleichung die singuläre Auflösung gesucht, ergiebt sich:

(1) $\left(\xi^{2}+v^{2}\right)\left(1-e^{2}\right)=4 e p \xi+4 p^{2}$.

Diese Gleichung gehört im Allgemeinen einem Kreise zu, dessen Mittelpunct in der Axe der $x$ liegt, oder in der gröfsern Hauptaxe der Linie der zweiten Ordnung, und zwar, wie sich durch eine sehr einfache Transformation der Coordinaten zeigt, in dem zweiten Brennpuncte dieser Linie der zweiten Ordnung. Diesen Kreis wollen wir für eine jede Curve insbesondere, die sich zu den Linien der zweiten Ordnung zählt, betrachten.

I. Wenn die Linie der zweiten Ordnung ein Kreis ist, so fallen beide Brennpuncte zusammen, und die Curve der Directrixen mufs ein concentrischer Kreis sein. Setzt man nemlich in der letzten Gleichung $e=0$, so stellt $p$ den Halbmesser des gegebenen Kreises vor, und die Curve der Directrixen wird dann durch folgende Gleichung:

$$
\xi^{2}+v^{2}=4 p^{2}
$$

vorgestellt, also ein concentrischer Kreis von doppelt so grofsem Halbmesser, welches die Natur der Sache ganz mit sich bringt.

II. Die Linie der zweiten Ordnung sei eine Parabel; da liêse sich ebenfalls sogleich die Gerade als Curve der Directrix vermuthen; dena der zweite Brennpunct der Parabel befindet sich in einer unendlichen Entfernung, daher die Krümmung des Kreises, welcher die Curve der Directrixen vorstellen soll, unendlich grofs sein mufs, oder eine Gerade. Setzt man nun in der vorletzten Gleichung $e=1$, so hat man:

$$
\xi=-p
$$

als Gleichung der Curve der Directrixen einer Parabel. Diese Gleichung stellt eine auf der Liauptaxe der Parabel senkrechte Gerade vor. Diese Gerade fällt wegen des negativen Zeichens aufserhalb der Parabel in dem Abstande $p$ von dem Brennpuncte, oder in dem Abstande $\frac{p}{2}$ von dem Scheitel der Parabel, welches bekannt ist.

III. Die Curve der zweiten Ordnung sei endlich eine Ellipse oder Hyperbel. Dann stellt die Gleichung (1), oder

$$
\xi^{2}+v^{2}=\frac{4 e p}{1-e^{2}} \xi+\frac{4 p^{2}}{1-e^{2}}
$$

die Curve der Directrixen vor. Da aber die Ellipse sowohl als die $\mathrm{Hy}_{\mathrm{y}}$ perbel zwei Brennpuncte hat, so haben diese Curven der zweiten Ordnung zwei Curven der Directrixen, welche Kreise sind, deren Mittelpunate

Crelle's Journal. II. Bd. 4. Hft: 
die Brennpuncte sind, und deren Radien, wie leicht zu sehen, gleich den grofsen Axen der Ellipsen oder Hyperbeln sind.

Diese hier auf analytischem Wege gefundenen Resultate, lassen sich noch leichter auf synthetischem Wege zeigen; welches wir aber hier übergehen.

Aus dem vorhin gewählten besonderen Falle sieht man, dafs sämmtliche Curven der zweiten Ordnung, wenn einer ihrer Brennpuncte der fixe Punct ist; Curven der Directrixen zulassen, wie bei der Parabel. Meines Wissens war eine solche Curve der Directrixen, unter dem einfachen Namen der Directrix, nur bei der Parabel bekannt, und die übrigen Linien der zweiten Ordnung scheinen von dieser Eigenschaft ausgeschlossen zu sein. Durch das so eben Gezeigte sieht man, dafs die Gerade die bei der Parabel die Eigenschaften der Curve der Directrixen hat, nur ein besonderer Fall eines Kreises ist, dessen Halbmesser für die Parabel unendlich grofs ist, und hiermit theilen sämmtliche Linien der zweiten Ordnung diese Eigenschaft, eine Curve der Directrixen zu haben.

Mit Hülfe einer solchen Curve der Directrixen lassen sich sämmtliche Linien der zweiten Ordnung zeichnen, wovon man sich leicht überzeugen kann.

Ganz auf demselben. Wege findet man, dafs die Curve der Directrixen der Linien der zweiten Ordnung mit einem Mittelpuncte, wenn der Mittelpunct der fixe Punct ist, durch folgende Gleichung:

$$
\left(v^{2}+\xi^{2}\right)^{2}=4\left(a^{2} \xi^{2}+b^{2} v^{2}\right)
$$

gegeben ist, wo $a$ und $b$ die halbe grofse und halbe kleine Axe der Linien der zweiten Ordnung vorstellen.

Da es keine Schwierigkeit haben würde, für jede beliebige Curve und irgend einen fixen Punct die Curve der Directrixen zu finden, so halte ich mich mit Aufführung mehrerer anderer besonderer Fälle nicht auf. 